\title{
ÉPOCAS DE INDUÇÃO FLORAL E SOMA TÉRMICA DO PERÍODO DO FLORESCIMENTO À COLHEITA DE ABACAXI 'SMOOTH CAYENNE'
}

\author{
SERGIO LUIZ COLUCCI DE CARVALHO², CARMEN SILVIA VIEIRA JANEIRO NEVES³, RODRIGO BÜRKLE4, \\ CELSO JAMIL MARUR ${ }^{2}$
}

\begin{abstract}
RESUMO - Na produção do abacaxi, é importante conhecer o efeito do clima no ciclo da cultura. O objetivo do trabalho foi estudar as somas térmicas do período da indução floral à colheita de abacaxi 'Smooth Cayenne' para diferentes épocas de indução floral no Norte do Paraná e sua influência na produção e qualidade dos frutos. Os tratamentos foram indução floral artificial com ethephon, nos meses de abril, maio, junho e julho, além da testemunha, induzida naturalmente. A inflorescência surgiu aos 115,5; 107,3; 77,3 e 48,3 dias após a indução floral, respectivamente, para os tratamentos de abril, maio, junho e julho. O período entre o surgimento da inflorescência e a colheita foi de 163 dias para a indução de abril, maior do que o observado para os demais tratamentos (144 a 151 dias). O tratamento de abril proporcionou frutos menores do que o de julho, mas a acidez titulável, o teor de sólidos solúveis e o "ratio" não foram influenciados pelas épocas de aplicação do indutor. A soma térmica média do período entre o florescimento e a colheita foi de 1.090 graus-dia, sem diferenças estatísticas entre as épocas de indução floral.
\end{abstract}

Termos para indexação: Ananas comosus, graus-dia, ethephon, brix.

\section{FLORAL INDUCTION PERIOD AND THERMAL TIME REQUIREMENTS FROM THE FLOWERING TO THE HARVEST PERIOD FOR SMOOTH CAYENNE PINEAPPLE}

\begin{abstract}
The knowledge of climate effects is important in planning pineapple production. The aim of this research was to accomplish the thermal time requirements of 'Smooth Cayenne' pineapple from the floral induction to the harvest period for different periods of artificial floral induction at Northern State of Paraná and also its influence in yield and fruit quality. The treatments were floral induction with ethephon in April, May, June and July. The control had natural induction. The inflorescence arose at 115.5; 107.3; 77.3 and 48.3 days after the floral induction for the applications in April, May, June, and July, respectively. The period between inflorescence arisen and harvest was 163 days for April induction, which was higher than for the other treatments (144 - 151 days). The fruits from the April treatment were smaller than the fruits from July. Period of floral induction had no effect on titratable acidity, total soluble solids and ratio of the fruits. The thermal time requirement from the flowering to the harvest period was 1,090 degree-days, with no statistical difference among treatments.
\end{abstract}

Index terms: Ananas comosus, degree-days, ethephon, brix.

\section{INTRODUÇÃO}

O abacaxizeiro é uma planta de origem tropical; o ciclo da cultura pode variar de 12 a mais de 24 meses, dependendo das condições climáticas, principalmente da temperatura, cuja faixa ótima para o crescimento das raízes e das folhas se situa entre 22 e $32^{\circ} \mathrm{C}$ (Py et al., 1984). Uma vez que a planta tenha alcançado tamanho suficiente para que ocorra a indução floral, fatores ambientais que promovem o florescimento são os mesmos que resultam em menores taxas de crescimento vegetativo, tais como diminuição do suprimento de água e nutrientes, reduzidos regimes de temperaturas, comprimento do dia e radiação solar (Bartholomew \& Kadzimann, 1975).

Py et al. (1984) relataram que o abacaxizeiro é uma planta de dias curtos e que é muito variável o tempo que leva para entrar na fase de diferenciação floral, ao passo que varia muito pouco o tempo compreendido entre tal fase e a maturação do fruto.

Em uma plantação de abacaxi, é desejável que a diferenciação floral ocorra simultaneamente em todas as plantas de um mesmo talhão, o que pode ser conseguido com a indução floral artificial. Assim, a adoção desta prática pode resultar em diminuição dos custos e escalonamento da colheita do abacaxi, racionalizando o uso da mão-deobra e ofertando frutos em períodos de escassez no mercado. O ethephon tem sido o produto mais utilizado para a indução floral do abacaxi, principalmente por sua praticidade. O período de colheita pode estenderse por mais de 60 dias quando não é feita a indução artificial do florescimento, e é de aproximadamente 15 dias quando essa prática é adotada (Cunha, 1999).

O ciclo das culturas, usualmente expresso em unidade de tempo, pode ser contabilizado em unidades de calor que possibilitem o desenvolvimento (Ometto, 1981). Assim, soma térmica por um período determinado, expressa em graus-dia, é o acúmulo da temperatura média diária subtraindo-se a temperatura-base, acima da qual a planta consegue desempenhar suas funções fisiológicas. Alfonsi (1994), para abacaxi cvs. Smooth Cayenne e Rondon, obteve as temperaturas-base de 9 e $5^{\circ} \mathrm{C}$ e as somas térmicas de 2.300 e 2.020 graus-dia do período de florescimento à colheita para as duas cultivares, respectivamente.

Conhecendo-se as condições climáticas regionais e o período entre a indução da florada e a colheita, sendo este período determinado pela soma térmica, pode-se planejar e escalonar a produção através da época de indução artificial da floração. Assim, este trabalho teve como objetivos determinar as exigências térmicas do período de indução floral à colheita e avaliar a influência da época de indução floral na produção, na época de colheita e na qualidade dos frutos de abacaxi 'Smooth Cayenne' no Norte do Paraná.

\section{MATERIAL E MÉTODOS}

O experimento foi conduzido em Londrina, no Instituto Agronômico do Paraná (IAPAR), em Latossolo Vermelho distroférrico, a $23^{\circ} 23^{\prime} \mathrm{S}$ e $566 \mathrm{~m}$ de altitude. Foram utilizadas mudas tipo filhote, da cultivar Smooth Cayenne, com 15 a $20 \mathrm{~cm}$ de altura, tratadas por imersão durante cinco minutos, em solução de $0,1 \%$ de ingrediente ativo de parathion metílico e $0,2 \%$ de ingrediente ativo de benomil. As mudas foram plantadas em 6 de outubro de 2000, em linhas duplas, no espaçamento de $1,00 \times 0,40 \times 0,40 \mathrm{~m}$. A adubação foi realizada mensalmente com $10 \mathrm{~g}$ de uréia e $10 \mathrm{~g}$ de cloreto de potássio por planta, de dezembro de 2000 a agosto de 2001 , e $15 \mathrm{~g}$ de cloreto de potássio por planta, de setembro de 2001 a março de 2002. Para o controle da brocado-fruto (Thecla basalides), foram realizadas aplicações quinzenais de parathion metílico a $0,1 \%$ de ingrediente ativo, no período de julho a

\footnotetext{
(Trabalho 076/2005). Recebido: 06/05/2005. Aceito para publicação: 04/11/2005.

2 Eng. Agr., PhD, Pesquisador, Instituto Agronômico do Paraná (IAPAR), C. P. 481, CEP 86001-970 - Londrina, PR. E-mail: slccarva@iapar.br.

${ }^{3}$ Eng $^{\mathrm{a}} \mathrm{Agr}^{\mathrm{a}}$, Dr ${ }^{\mathrm{a}}$, Professora, Universidade Estadual de Londrina (UEL), Departamento de Agronomia, C. P. 6001 - CEP 86051-990 - Londrina, PR. Bolsista CNPq.Email: csvjneve@uel.br.

${ }^{4}$ Eng. Agr., Mestrando em Agronomia, UEL - Departamento de Agronomia, C. P. 6001 - CEP 86051-990 - Londrina, PR. E-mail: burkle.rod@bol.com.br.
} 
outubro de 2002

Os tratamentos de indução floral constituíram-se da aplicação de $50 \mathrm{ml}$ por planta de solução de ethephon a $1.000 \mathrm{ppm}$, no primeiro dia dos meses de abril, maio, junho e julho de 2002, sendo a testemunha o tratamento sem indução floral artificial. O delineamento experimental foi o de blocos casualizados, com cinco tratamentos, seis repetições e 30 plantas úteis por parcela.

As aplicações foram realizadas entre $8 \mathrm{~h} 30$ e 9 horas da manhã, em condições de tempo estável. Considerou-se o florescimento quando $50 \%$ das plantas haviam emitido a inflorescência, observando-se as plantas que apresentavam a inflorescência visível no fundo da roseta foliar. Os frutos foram cobertos com papel, quando do secamento das pétalas, e colhidos quando apresentaram o terço basal da casca amarelado, de 16 de dezembro de 2002 a 28 de janeiro de 2003. Registrouse semanalmente o número de frutos colhidos por tratamento, e a determinação da data da colheita foi feita por média ponderada. Foram feitas determinações de peso do fruto com e sem coroa, teor de sólidos solúveis (SS), acidez total titulável (ATT) do suco, pelos métodos da AOAC (1990) e ratio (SS/ATT).

Foi observado o número de dias entre o plantio, a indução floral, o florescimento e a colheita. Para cada período avaliado, foram calculadas as constantes térmicas em graus-dia (GD), com a fórmula $\mathrm{GD}=\mathrm{a}\left(\mathrm{T}_{\mathrm{a}}-\mathrm{T}_{\mathrm{b}}\right)$, utilizando-se da temperatura base $\left(\mathrm{T}_{\mathrm{b}}\right)$ de $15,8^{\circ} \mathrm{C}$, determinada por Carvalho et al. (2000). A temperatura média diária compensada $\left(\mathrm{T}_{\mathrm{a}}\right)$ foi obtida na Área de Agrometeorologia do IAPAR, somando-se as temperaturas máxima, mínima, temperatura às $9 \mathrm{~h}$ e temperatura às $21 \mathrm{~h}$, sendo a última multiplicada por 2 , e a soma dividida por 5 (Ometto, 1981). Os dados foram submetidos à análise de variância, complementada pelo teste de Tukey.

\section{RESULTADOS E DISCUSSÃO}

O tratamento de indução floral, em abril, apresentou florescimento em meados de julho, enquanto os demais tratamentos tiveram florescimento no início de agosto (Figura 1). No dia 8 de agosto de $2002,87,5 \%$ das plantas do tratamento de abril apresentaram inflorescência, enquanto nas induzidas em maio e junho isto ocorreu em $15 \%$ e $10,4 \%$ delas, respectivamente; e nos tratamentos de julho e testemunha, a emissão da inflorescência atingiu valores menores que $10 \%$. A partir daí, todos os tratamentos apresentaram aumento rápido da taxa de emissão de inflorescências, com exceção do tratamento de abril, que já havia atingido taxa elevada. Em 16 de agosto, a taxa do tratamento de abril continuou inalterada, enquanto a dos demais tratamentos aumentou para cerca de 40\%. Em 30 de agosto, as percentagens de plantas com inflorescência foram superiores a $90 \%$ em todos os tratamentos. Os resultados sugerem que a diferenciação floral nas plantas tratadas em junho, julho e a indução natural devem ter ocorrido em datas muito próximas. A indução realizada em maio, embora tenha resultado na presença de algumas inflorescências emitidas nas primeiras avaliações, apresentou, nas avaliações subseqüentes, comportamento similar ao dos tratamentos realizados posteriormente. Estes resultados são semelhantes aos obtidos por Auler et al. (2002) no Noroeste do Paraná e por Giacomelli et al. (1979) no Planalto Paulista, que constataram a ocorrência de diferenciação floral espontânea do abacaxizeiro 'Smooth Cayenne' no outono, a partir de maio. No presente trabalho, os tratamentos de indução floral, em abril, maio, junho e julho, resultaram em taxa acumulada de aparecimento de inflorescência em 50 $\%$ das plantas, aos 122; 111; 87 e 51 dias após a aplicação da solução, respectivamente (Tabela 1). Spironello et al. (1997) verificaram a ocorrência de emissão floral natural aos 105 dias após um período de queda de temperatura.

O período do surgimento da inflorescência (florescimento) à colheita variou de 144,0 a 163,1 dias (Tabela 1), sendo significativamente superior para o tratamento de abril em relação aos demais. Estes valores ficaram acima dos 136,5 dias observados em Votuporanga-SP (Spironello et al., 1997). Comparando-se a amplitude dos dois períodos, a diferença

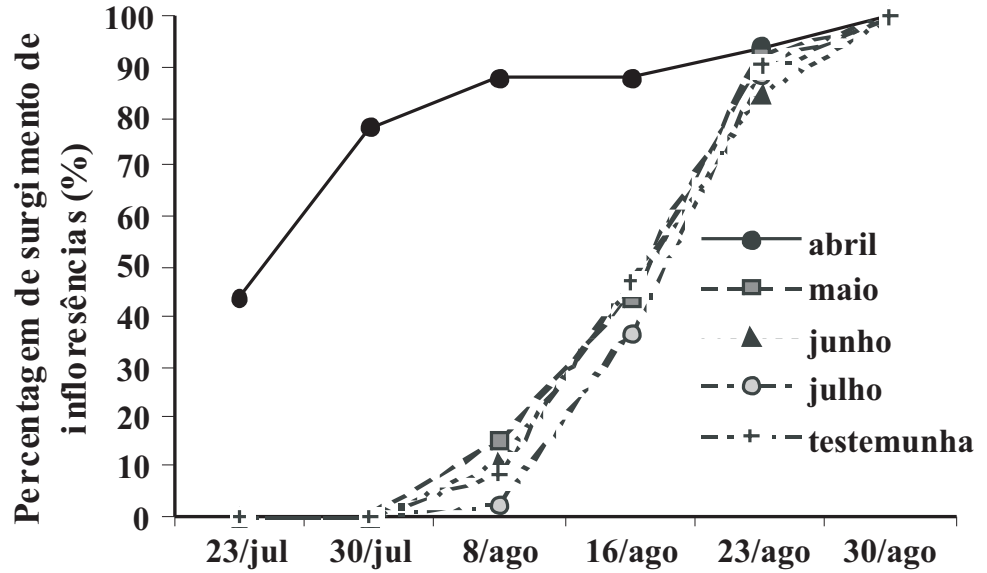

FIGURA 1 - Datas de ocorrência e percentagem acumulada de surgimento das inflorescências em plantas de abacaxizeiro 'Smooth Cayenne' submetido a diferentes épocas de indução floral. Londrina -PR, 2002.

TABELA 1 - Número de dias do plantio à indução floral, da indução ao surgimento de $50 \%$ das inflorescências (florescimento), do florescimento à colheita, da indução à colheita e do plantio à colheita de plantas de abacaxizeiro 'Smooth Cayenne' submetido a diferentes épocas de indução floral. Londrina-PR, 2002.

\begin{tabular}{lccccl}
\hline Tratam. & $\begin{array}{c}\text { Plantio- } \\
\text { Indução }\end{array}$ & $\begin{array}{c}\text { Indução- } \\
\text { Florescim. }\end{array}$ & $\begin{array}{c}\text { Florescim.- } \\
\text { Colheita }\end{array}$ & $\begin{array}{c}\text { Indução- } \\
\text { Colheita }\end{array}$ & $\begin{array}{c}\text { Plantio- } \\
\text { Colheita }\end{array}$ \\
\cline { 2 - 6 } & \multicolumn{5}{c}{ dias } \\
\hline Abril & 543 & $115,6 \mathrm{a}$ & $163,1 \mathrm{a}$ & $278,8 \mathrm{a}$ & $821,7 \mathrm{~b}$ \\
Maio & 573 & $107,3 \mathrm{~b}$ & $144,0 \mathrm{~b}$ & $251,3 \mathrm{~b}$ & $826,0 \mathrm{ab}$ \\
Junho & 604 & $77,3 \mathrm{c}$ & $147,2 \mathrm{~b}$ & $224,5 \mathrm{c}$ & $828,5 \mathrm{ab}$ \\
Julho & 634 & $48,3 \mathrm{~d}$ & $147,8 \mathrm{~b}$ & $196,2 \mathrm{~d}$ & $830,2 \mathrm{a}$ \\
Testem. & - & - & $151,2 \mathrm{~b}$ & - & $831,2 \mathrm{a}$ \\
\hline
\end{tabular}

"Médias seguidas pela mesma letra, na coluna não diferem entre si, pelo teste de Tukey, a 5\% de probabilidade.

entre o tratamento que mais adiantou o ciclo (abril) e o que menos adiantou (julho), foi de 67 dias para o período indução-florescimento e de apenas 19 dias para o período florescimento-colheita, indicando que o primeiro período é mais sensível às variações climáticas do que o segundo.

Mesmo sendo o período do florescimento à colheita para o tratamento de abril maior do que o observado para os demais tratamentos, houve uma antecipação de até 15 dias na colheita, pois, no dia 2 de janeiro, já haviam sido colhidos $68 \%$ dos frutos das plantas induzidas em abril, enquanto as induzidas nas demais épocas, os valores superiores a 70\% só foram atingidos em 17 de janeiro (Tabela 2). Esta antecipação pode ser interessante para o produtor, considerando que os frutos entrariam no mercado antes do período de maior oferta. Também houve

TABELA 2 - Percentagem acumulada, em diferentes datas, de frutos colhidos de abacaxi 'Smooth Cayenne' submetido a diferentes épocas de indução floral. Londrina-PR, 20022003.

\begin{tabular}{ccrrrrr}
\hline & \multicolumn{6}{c}{ Épocas de indução floral } \\
\cline { 2 - 6 } Datas & Abril & Maio & Junho & Julho & Testem. & C.V. (\%) \\
\hline 16/dez & 0,0 a & $9,3 \mathrm{a}$ & $0,0 \mathrm{a}$ & $0,0 \mathrm{a}$ & $0,0 \mathrm{a}$ & 547,7 \\
26/dez & $37,8 \mathrm{a}$ & $11,6 \mathrm{ab}$ & $0,0 \mathrm{~b}$ & $0,0 \mathrm{~b}$ & $0,0 \mathrm{~b}$ & 159,7 \\
2/jan & $68,2 \mathrm{a}$ & $26,5 \mathrm{~b}$ & $25,7 \mathrm{~b}$ & $8,2 \mathrm{~b}$ & $5,4 \mathrm{~b}$ & 54,5 \\
10/jan & $78,8 \mathrm{a}$ & $47,2 \mathrm{~b}$ & $53,0 \mathrm{ab}$ & $55,9 \mathrm{ab}$ & $46,5 \mathrm{~b}$ & 24,9 \\
17/jan & $93,4 \mathrm{a}$ & $75,3 \mathrm{a}$ & $79,7 \mathrm{a}$ & $83,0 \mathrm{a}$ & $85,7 \mathrm{a}$ & 17,8 \\
20/jan & $94,5 \mathrm{a}$ & $99,3 \mathrm{a}$ & $95,3 \mathrm{a}$ & $96,8 \mathrm{a}$ & $96,3 \mathrm{a}$ & 9,8 \\
28/jan & $95,1 \mathrm{a}$ & $100,0 \mathrm{a}$ & $100,0 \mathrm{a}$ & $100,0 \mathrm{a}$ & $100,0 \mathrm{a}$ & 7,4 \\
6/fev & $100,0 \mathrm{a}$ & - & - & - & - & 0,0 \\
\hline
\end{tabular}

*Médias seguidas pela mesma letra, na linha, não diferem entre si, pelo teste de Tukey, a $5 \%$ de probabilidade. 
um maior escalonamento do período de colheita, o que pode facilitar a racionalização da mão-de-obra, embalagem, transporte e distribuição do produto, diminuindo custos na pequena propriedade. Por outro lado, a região está sujeita à ocorrência de geada, que pode causar danos às inflorescências obtidas com a indução precoce, como relatado por Auler et al. (2002) para as induções realizadas em março e abril de 2000 no Noroeste do Paraná. O ciclo total da cultura variou de 821,7 a 831,2 dias (Tabela 1).

O tratamento de julho apresentou maior peso médio dos frutos do que o de abril (Tabela 3). Como todas as plantas tinham a mesma idade, as induzidas em julho tiveram maior período vegetativo, o que pode ter contribuído para um maior acúmulo de reservas a serem carreadas aos frutos, o que também foi observado em outros trabalhos (Bezerra et al., 1979; Cunha et al., 1993). Os teores de acidez titulável e de sólidos solúveis, além de "ratio", não apresentaram diferenças significativas entre os tratamentos, ao contrário de outros trabalhos em que foram encontrados frutos mais ácidos e com menor teor de sólidos solúveis em induções mais precoces (Bezerra et al., 1979; Choairy, 1983).

TABELA 3 - Peso do fruto com coroa, peso da coroa, teor de sólidos solúveis, acidez titulável e 'ratio' dos frutos de abacaxizeiro 'Smooth Cayenne' submetido a diferentes épocas de indução floral. Londrina-PR, 2002-2003.

\begin{tabular}{|c|c|c|c|c|c|}
\hline \multirow[t]{2}{*}{ Tratam. } & $\begin{array}{l}\text { Peso } \\
\text { fruto }\end{array}$ & $\begin{array}{c}\text { Peso } \\
\text { Coroa }\end{array}$ & $\begin{array}{l}\text { Sólidos } \\
\text { solúveis }\end{array}$ & Acidez & 'Ratio' \\
\hline & \multicolumn{2}{|c|}{ (g) } & (brix) & $\begin{array}{c}(\% \\
\text { ác.citr. })\end{array}$ & \\
\hline Abril & $1.537,2 \mathrm{~b}^{*}$ & $236,1 \mathrm{ab}$ & $15,0 \mathrm{a}$ & $0,61 \mathrm{a}$ & $24,8 \mathrm{a}$ \\
\hline Maio & $1.604,4 \mathrm{ab}$ & $244,5 \mathrm{ab}$ & $15,7 \mathrm{a}$ & $0,66 \mathrm{a}$ & $23,8 \mathrm{a}$ \\
\hline Junho & $1.744,6 \mathrm{ab}$ & 283,7 a & $15,3 \mathrm{a}$ & $0,74 \mathrm{a}$ & $20,7 \mathrm{a}$ \\
\hline Julho & $1.808,9 \mathrm{a}$ & $235,2 \mathrm{ab}$ & $14,8 \mathrm{a}$ & $0,63 \mathrm{a}$ & $23,5 \mathrm{a}$ \\
\hline Testem & $1.617,7 \mathrm{ab}$ & $205,5 \quad b$ & $14,9 \mathrm{a}$ & $0,64 \mathrm{a}$ & $23,4 \mathrm{a}$ \\
\hline
\end{tabular}

"Médias seguidas pela mesma letra, na coluna, não diferem entre si, pelo teste de Tukey, a $5 \%$ de probabilidade.

A soma térmica do período indução-florescimento variou de 104,8 a 519,6 graus-dia, com diferenças significativas entre todos os tratamentos (Tabela 4). Quanto mais tardia a indução, menor foi a soma térmica observada. O conceito do acúmulo de graus-dia pressupõe um modelo em que os demais fatores climáticos permanecem constantes. Entretanto, ao se efetuar os tratamentos de indução em diferentes épocas, em condições de campo, certamente outros fatores climáticos além da soma térmica das temperaturas do ar também variaram, como o comprimento do dia, a temperatura do solo, a disponibilidade hídrica, entre outros, o que pode auxiliar a explicar as diferenças encontradas na soma térmica entre os tratamentos, pois estes outros fatores também podem afetar o ciclo das plantas, como observado por Almeida et al. (2002), que tiveram redução do ciclo em plantas irrigadas quando comparadas com plantas não irrigadas no mesmo período e, portanto, submetidas às mesmas temperaturas do ar.

Para o período entre o florescimento e a colheita, não houve diferenças significativas entre os tratamentos, sendo que as diferenças observadas para o período total entre a aplicação da solução indutora e a colheita foram determinadas, basicamente, pelas diferenças observadas no período que antecede o aparecimento da inflorescência. Obteve-se a soma térmica média de 1.090 graus-dia entre o florescimento e a colheita (Tabela 4). Este resultado difere daquele obtido por Alfonsi (1994) em São Paulo, que observou 2.300 graus-dia para o mesmo período. Esse autor, no entanto, considerou $9^{\circ} \mathrm{C}$ como temperaturabase, enquanto no presente trabalho utilizou-se da temperatura-base de $15,8^{\circ} \mathrm{C}$, calculada por Carvalho et al. (2000). Caso fosse considerada a mesma temperatura-base adotada por Alfonsi (1994), a soma térmica teria sido de 2.040 graus-dia, muito mais próxima da encontrada por aquele autor. $\mathrm{O}$ trabalho foi realizado praticamente entre o equinócio de primavera a meados do verão, quando há aumento acentuado do
TABELA 4 - Soma térmica das fases de plantio à indução floral, da indução ao surgimento de $50 \%$ das inflorescências (florescimento), do florescimento à colheita, da indução à colheita e do plantio à colheita do abacaxizeiro 'Smooth Cayenne' submetido a diferentes épocas de indução floral. Londrina-PR, 2002-2003.

\begin{tabular}{lccccc}
\hline Tratam. & $\begin{array}{c}\text { Plantio- } \\
\text { Indução }\end{array}$ & $\begin{array}{c}\text { Indução- } \\
\text { Florescim. }\end{array}$ & \begin{tabular}{c} 
Florescim.- \\
Colheita \\
\cline { 2 - 6 }
\end{tabular} & $\begin{array}{c}\text { Indução- } \\
\text { Colheita }\end{array}$ & $\begin{array}{c}\text { Plantio- } \\
\text { Colheita }\end{array}$ \\
\hline Abril & $3.516,5$ & $518,6 \mathrm{a}^{*}$ & $1.100,0 \mathrm{a}$ & $1.618,7 \mathrm{a}$ & $5.128,9 \mathrm{~b}$ \\
Maio & $3.784,8$ & $328,0 \mathrm{~b}$ & $1.061,3 \mathrm{a}$ & $1.389,3 \mathrm{~b}$ & $5.168,0 \mathrm{ab}$ \\
Junho & $3.900,4$ & $215,0 \mathrm{c}$ & $1.082,9 \mathrm{a}$ & $1.297,9 \mathrm{c}$ & $5.195,1 \mathrm{ab}$ \\
Julho & $4.018,5$ & $104,8 \mathrm{~d}$ & $1.091,7 \mathrm{a}$ & $1.196,5 \mathrm{~d}$ & $5.210,2 \mathrm{a}$ \\
Testem. & - & - & $1.114,1 \mathrm{a}$ & - & $5.217,8 \mathrm{a}$ \\
Média & - & - & $1.090,0$ & - & - \\
\hline
\end{tabular}

"Médias seguidas pela mesma letra, na coluna, não diferem entre si, pelo teste de Tukey, a $5 \%$ de probabilidade.

fotoperíodo para a região. Trabalhos futuros nessa linha poderiam incluir o comprimento do dia nos modelos.

\section{CONCLUSÕES}

1. No Norte do Paraná, a indução artificial do florescimento do abacaxizeiro 'Smooth Cayenne', feita em abril, antecipa a emissão da inflorescência e a colheita em até 15 dias em relação às induções feitas em maio e junho.

2. No período da emissão da inflorescência à colheita, o abacaxizeiro 'Smooth Cayenne' apresenta soma térmica de 1.090 grausdia, considerando-se a temperatura-base de $15,8^{\circ} \mathrm{C}$.

3. O peso médio dos frutos é menor para a época de indução mais precoce.

\section{REFERÊNCIAS}

ALFONSI, R. R. Temperatura-base e graus-dias para duas variedades de abacaxizeiro em São Paulo. In: CONGRESSO BRASILEIRO DE FRUTICULTURA, 13., 1994, Salvador. Resumos... Salvador: SBF, 1994.p.51-52

ALMEIDA, O.A.; SOUZA, L.F.S.; REINHARDT, D.H.; CALDAS, R.C. Influência da irrigação no ciclo do abacaxizeiro cv. Pérola em área de tabuleiro costeiro da Bahia. Revista Brasileira de Fruticultura, Jaboticabal, v.24, n.2, p.431-435, 2002.

AOAC-Association of Official Agricultural Chemists. Official methods of analysis of the association of the agricultural chemistry. $15^{\text {th. }}$ ed. Washington: AOAC, 1990. 1.015p.

AULER, P.A.; CARVALHO, S.L.C.; MARUR, C.J.; GOMES, J.C. Efeito de épocas de ethephon sobre a antecipação do florescimento do abacaxizeiro Smooth Cayenne na região Noroeste do Paraná. In: CONGRESSO BRASILEIRO DE FRUTICULTURA,17.,2002. Belém. Anais... Belém: SBF, 2002. CD-Rom.

BARTHOLOMEW, D.P.; KADZIMANN, S.B. Eco-physiology of Pineapple. In: SIMPÓSIO SOBRE ECOFISIOLOGIADECULTIVOS TROPICAIS, 1975. Manaus.

BEZERRA, J.E.F.; LEDERMAN, I.E.; AGUILAR, J.A.E.; REIS, O.V. Influência da idade de indução do florescimento e do peso dos filhotes sobre a produção e qualidade do abacaxizeiro 'Cayenne'. In: CONGRESSO BRASILEIRO DE FRUTICULTURA, 5., 1979, Pelotas. Anais... Pelotas: SBF, 1979. v.1, p. 327-338.

CARVALHO, S.L.C.; BÜRKLE, R.; LUDOVICO, D. Determinação da temperatura-base do abacaxizeiro cultivares Smooth Cayenne e Pérola. In: CONGRESSO BRASILEIRO DE FRUTICULTURA, 16., 2000, Fortaleza. Anais... Fortaleza: SBF, 2000. CD ROM.

CHOAIRY, S.A. Época de indução do florescimento, rendimento e qualidade do abacaxi. Pesquisa Agropecuária Brasileira, Brasília, v.18, n.3, p. 249-252, 1983. 
CUNHA, G.A.P.; REINHARDT, D.H.; CALDAS, R.C. Efeito da época de plantio, tamanho da muda e idade da planta na indução floral sobre o rendimento do abacaxizeiro 'Pérola' na Bahia. Revista Brasileira de Fruticultura, Cruz das Almas, v.15, n.3, p.43-50, 1993.

CUNHA, G.A.P. Florescimento e uso de fitorreguladores. In: CUNHA, G.A.P.; CABRAL, J.R.S.; SOUZA, L.F.S. O abacaxizeiro: cultivo, agroindústria e economia. Brasília: Embrapa, 1999. p. 229-251

GIACOMELLI, E.J.; PY, C.; LOSSOIS, P. Estudo sobre épocas de produção para o abacaxizeiro Cayenne, no planalto paulista. In:
CONGRESSO BRASILEIRO DE FRUTICULTURA, 5., 1979, Pelotas. Anais... Pelotas: SBF, 1979. v.2, p. 499-511.

OMETTO, J. C. Bioclimatologia vegetal. São Paulo: Ceres, 1981. 400p. PY, C.; LACOEUILHE, J. J.; TEISSON, C. L’ Ananás: sa culture, ses produits. Paris: Maisonneuve et Larose, 1984. 537p.

SPIRONELLO, A.; BORTOLETTO, N.; SIGRIST, J.M.M.; NAGAI, V. Avaliação agrotecnológica e do ciclo de variedades de abacaxizeiro, em duas densidades, em Votuporanga (SP). Bragantia, Campinas, v.56, n.2, p.343-355, 1997. 